\section{RMD Open}

Rheumatic \& Musculoskeletal Diseases

\title{
Fractal analysis of perfusion imaging in synovitis: a novel imaging biomarker for grading inflammatory activity based on assessing angiogenesis
}

To cite: Michallek F, Ulas ST, Poddubnyy D, et al. Fractal analysis of perfusion imaging in synovitis: a novel imaging biomarker for grading inflammatory activity based on assessing angiogenesis. RMD Open 2022;8:e002078. doi:10.1136/ rmdopen-2021-002078

Received 3 November 2021 Accepted 17 January 2022

\section{Check for updates}

(c) Author(s) (or their employer(s)) 2022. Re-use permitted under CC BY-NC. No commercial re-use. See rights and permissions. Published by BMJ.

${ }^{1}$ Department of Radiology, Charité - Universitätsmedizin Berlin, corporate member of Freie Universität Berlin and Humboldt- Universität zu Berlin Berlin, Germany

${ }^{2}$ Department of

Gastroenterology, Infectiology and Rheumatology (including Nutrition Medicine), Charité - Universitätsmedizin Berlin, corporate member of Freie Universität Berlin and HumboldtUniversität zu Berlin, Berlin, Germany

${ }^{3}$ Department of Rheumatology, Charité - Universitätsmedizin Berlin, corporate member of Freie Universität Berlin and Humboldt- Universität zu Berlin, Berlin, Germany

Correspondence to Dr Florian Michallek; florian.michallek@charite.de

\section{ABSTRACT}

Objectives The mutual and intertwined dependence of inflammation and angiogenesis in synovitis is widely acknowledged. However, no clinically established tool for objective and quantitative assessment of angiogenesis is routinely available. This study establishes fractal analysis as a novel method to quantitatively assess inflammatory activity based on angiogenesis in synovitis.

Methods First, we established a pathophysiological framework for synovitis including fractal analysis of software perfusion phantoms, which allowed to derive explainability with a known and controllable reference standard for vascular structure. Second, we acquired MRI datasets of patients with suspected rheumatoid arthritis of the hand, and three imaging experts independently assessed synovitis analogue to Rheumatoid Arthritis MRI Scoring (RAMRIS) criteria Finally, we performed fractal analysis of dynamic firstpass perfusion MRI in vivo to evaluate angiogenesis in relation to inflammatory activity with RAMRIS as reference standard.

Results Fractal dimension (FD) achieved highly significant discriminability for different degrees of inflammatory activity $(p<0.01)$ in software phantoms with known ground-truth of angiogenic structure. FD indicated increasingly chaotic perfusion patterns with increasing grades of inflammatory activity (Spearman's $\rho=0.94, p<0.001)$. In 36 clinical patients, fractal analysis quantitatively and objectively discriminated individual RAMRIS scores $(p \leq 0.05)$. Area under the receiveroperating curve was $0.84(95 \% \mathrm{Cl} 0.7$ to 0.89$)$ for fractal analysis when considering RAMRIS as ground-truth. Fractal analysis additionally identified angiogenesis in cases where RAMRIS underestimated inflammatory activity.

Conclusions Based on angiogenesis and perfusion pathophysiology, fractal analysis non-invasively enables comprehensive, objective and quantitative characterisation of inflammatory angiogenesis with subjective and qualitative RAMRIS as reference standard. Further studies are required to establish the clinical value of fractal analysis for diagnosis, prognostication and therapy monitoring in inflammatory arthritis.

\section{Key messages}

What is already known about this subject?

- Angiogenesis plays a pivotal role in the pathophysiology of synovitis in chronic inflammatory joint diseases both as a cause and a symptom of inflammation.

However, so far non-invasive quantification of vascular structural changes due to inflammatory angiogenesis is not available in clinical routine.

What does this study add?

- Fractal analysis of perfusion imaging allows noninvasive, quantitative and objective assessment of inflammatory activity in synovitis.

- Fractal dimension is a pathophysiological comprehensive imaging biomarker for inflammatory angiogenesis and can be implemented in perfusion MRI.

How might this impact on clinical practice or further developments?

- Fractal analysis might enable disease activity detection, thus facilitating early detection and disease activity evaluation during the follow-up in patients with rheumatoid arthritis.

\section{INTRODUCTION}

Angiogenesis plays an essential role in the pathophysiology of synovitis in chronic inflammatory joint diseases and is both symptom and a prerequisite for pathological synovial proliferation. ${ }^{1-3}$ However, clinical assessment methods do not provide quantitative, objective information on vascular structure in relation to inflammatory activity. Inflammatory hyperperfusion results in accelerated dynamics or increased uptake of contrast agent on contrast-enhanced MRI or CT, which has been correlated semiquantitatively to Doppler sonography assessment. ${ }^{4-7}$ Moreover, quantitative perfusion assessment using contrast agent kinetic models like the 
extended Tofts model has been previously proposed to quantify transfer constant $\mathrm{K}_{\text {trans }}$ and plasma volume $\mathrm{v}_{\mathrm{p}}{ }^{8-10}$ However, those semiquantitative or quantitative methods lack information on the underlying microvascular architecture. Also, the Rheumatoid Arthritis MRI Scoring System (RAMRIS) of the Outcome Measures in Rheumatology group, which is currently used for scoring imaging findings in inflammatory arthritis, does not account for comprehensive structural characterisation of angiogenesis. ${ }^{11}$

Angiogenesis is thought to constitute both symptom and promotor of synovial inflammation by providing surface area for inflammatory cell migration. ${ }^{1}$ Despite being hyperperfused, synovitis consistently involves tissue hypoxia. ${ }^{12}$ This paradoxical feature allows to infer the abnormal structure and function of the underlying vasculature, which has been investigated from macrovascular, microvascular and molecular perspectives. ${ }^{3} 1314$ Progressive vascular dedifferentiation by inflammatory angiogenesis might alter perfusion patterns towards higher geometrical complexity, which can be visualised using intravascular contrast agents. The vascular tree is statistically self-similar and scale-invariant and can thus be considered a fractal. ${ }^{15}$ This fractal organisation also applies to perfusion as functional correlate of vascular anatomy and can be quantified by fractal analysis using radiological imaging. ${ }^{16-18}$ Fractal analysis non-invasively evaluates changes in perfusion patterns and thus allows to infer alterations in vascular structure and function. Similar to tumours, angiogenesis in inflammatory arthritis is dysregulated with formation of abnormal vessel trees. ${ }^{19}$ The impact of dysregulated angiogenesis on perfusion is quantified by fractal dimension (FD) which reflects vascular chaos. ${ }^{15}$ Due to the close intertwining of angiogenesis and inflammatory activity with mutual promotion and alimentation, ${ }^{1}$ angiogenesis-induced hyperperfusion might be both a diagnostic and a therapeutic target. ${ }^{19} 20$
This study investigates fractal analysis of perfusion using MRI as an imaging biomarker for assessing angiogenesis in synovitis. A pathophysiological framework is formulated and validated using in silico phantoms based on microvascular tree models. Insights gained from those in silico experiments were transferred to a clinical patient population with inflammatory arthritis to conclude on vascular alterations induced by inflammation.

\section{METHODS}

In silico experiments

To investigate the pathophysiological hypothesis, we simulated vascular trees and perfusion patterns. This approached constituted a known and controllable reference standard of vascular structure and perfusion to derive explainability of fractal analysis. We build our models based on previously established, physiologically reasonable parametrisation and varied them systematically to approximate angiogenesis found under pathological conditions with different optimisation targets. ${ }^{21-26}$ Vascular models were composed of host trees representing normally perfused synovia, and interspersed angiogenic nests. Those angiogenic nests were generated to feature extensive endothelial surface area as found in inflammation. Those trees were progressively altered in microvascular density and perfusion rate to simulate progressive degrees of inflammatory activity. From those vascular models, perfusion phantoms were calculated (see figure 1). In total, three distinct degrees of inflammatory activity were simulated, and 10 perfusion phantoms per degree (30 phantoms in total) were subjected to fractal analysis.

\section{Generation of in silico models}

The constructive constrained optimisation algorithm ${ }^{27}$ was used to calculate the vascular tree models. A host
A

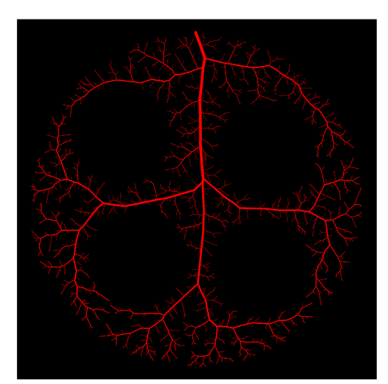

B Angiogenic nests with varying inflammatory activity

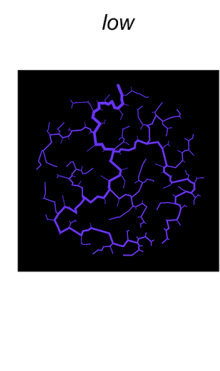

intermediate

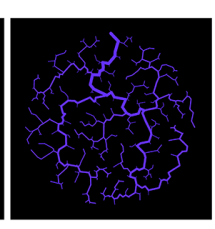

high

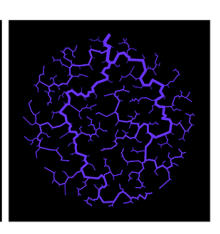

C Vasculature and perfusion phantom



Figure 1 Pathophysiological framework illustrated with in silico models. (A) Vascular tree model of non-inflamed, synovial host tissue with placeholders for four angiogenic nests. (B) Vascular tree models of angiogenic nests with alterations in vascular structure representing varying degrees of inflammatory activity. To simulate the changes in vascular structure and perfusion patterns induced by inflammation, vessel architecture in angiogenic nests is shifted from an optimality perspective: in healthy tissue, vascular structure is designed to minimise the work needed to generate, perfuse and maintain the vascular network. In inflammation, however, endothelial surface is increased to provide Gateways for migration of inflammatory cells into the inflamed tissue by altering vascular structure and increasing vascular density as well as perfusion rate. (C) Assembled vascular tree model of host and angiogenic nests together with the corresponding grey-level-encoded perfusion model in full resolution. Note the depiction of perfusion territories as a function of vascular scale. Before fractal analysis was performed, resolution was reduced by a factor of 0.01 . 
vascular tree representing normal synovial vasculature was calculated to minimise the physical work required to create, maintain and perfuse the tree. ${ }^{28}{ }^{29}$ The host tree perfused a circular area with a radius of $2 \mathrm{~cm}$ or 2000 pixels at a resolution of $10 \mu \mathrm{m}$ per pixel with 1500 capillary terminal locations and an assumed inlet flow rate of 10 arbitrary units (a.u.). The circular area was infiltrated by four approximately circular areas (radius $0.6 \mathrm{~cm}$ ) for insertion of inflammatory angiogenic nests. Thus, absolute perfusion rate in the host tree was $1.2 \mathrm{a} . \mathrm{u} . / \mathrm{cm}^{2}$. The inflammatory trees were optimised for their vascular length to provide a larger endothelial surface. To simulate progressive stages of inflammatory activity, vascular density in inflammatory nest trees (radius $0.6 \mathrm{~cm}$ or 600 pixels) was set to 250,375 or 500 terminal locations with inlet flow rates of 2, 3, or 4 a.u. (absolute perfusion rate $1.8,2.7$, or 3.5 a.u. $/ \mathrm{cm}^{2}$ ), respectively, to simulate low, intermediate and high grades of inflammatory activity. For all types of trees, perfusion pressure was $100 \mathrm{~mm} \mathrm{Hg}$, terminal pressure was $60 \mathrm{~mm} \mathrm{Hg}$, and bifurcation exponent was 3. Since our in silico models perfuse fictitious two-dimensional tissue, we based our quantitative simulations on a.u. within reasonable assumptions. ${ }^{30}$

The simulated vascular trees were used to calculate perfusion phantoms as follows. Based on the fractal structure of perfusion territories, the territory of each vascular segment was cumulatively simulated by the product of the FD determining quotient $\ln$ (relative blood flow) $/ \ln$ (relative area) times the actual perfusion rate. Original perfusion phantoms had a resolution of $10 \mu \mathrm{m}$ per pixel. To match the spatial resolution of clinical MRI, the original phantoms were reduced to a resolution of $1 \mathrm{~mm}$ per pixel, which constituted the final phantoms that were subjected to fractal analysis. In total, 10 phantoms were created for each of the three stages of inflammatory activity. Figure 1 summarises the pathophysiological framework and gives an illustration of the in silico phantoms.

\section{Patients and patient involvement}

Our study population constituted a convenience series and included patients, who were referred to the inpatient or outpatient clinic of rheumatology in our institution with suspected or known rheumatoid arthritis, the latter fulfilling the classification criteria of the American College of Rheumatology (ACR). ${ }^{31}$ We analysed data from prospectively recruited patients during a previous study at our institution from September 2016 to October 2017. ${ }^{52}$ Diagnosis was established by board-certified rheumatologists by clinical findings (history, swollen and tender joints), laboratory findings ( $\mathrm{C}$ reactive protein, rheumatoid factors, anticitrullinated protein antibodies) and imaging findings. No exclusion criteria applied other than age $>50$ years (due to a requirement of our local ethics committee) and absent contraindications to the MRI procedure. Patients were involved by systematically surveying their concerns and comfort with the MRI examination as previously detailed. ${ }^{532}$

\section{Magnetic resonance imaging}

Multiparametric MRI was performed on a 1.5 Tesla Magnetom Avanto scanner (Siemens, Erlangen, Germany) with a flexible 4-channel dedicated hand coil (Siemens, Erlangen, Germany). The protocol included the coronal acquisition of a T1-weighted and short-tau inversion recovery sequence. First-pass perfusion imaging was performed using a dynamic volume-interpolated breath-hold examination sequence with a temporal resolution of one volume per $10 \mathrm{~s}$ (scan parameters: $3 \mathrm{~mm}$ slice thickness, TE $2.53 \mathrm{~ms}$, TR $6.3 \mathrm{~ms}$, acquisition matrix $192 \times 128$ pixels) after intravenous administration of a weight-adapted dose of gadoterate (Dotarem, Guerbet, Roissy, France; $0.2 \mathrm{~mL} / \mathrm{kg}$ body weight) at a flow rate of $3 \mathrm{~mL} / \mathrm{s}$. Fractal analysis was performed using the firstpass perfusion sequences. RAMRIS scoring included a 3 min postcontrast T1-weighted fat-saturated sequence in coronal and axial orientation. Imaging protocol details were described previously. ${ }^{53}$

\section{Clinical evaluation and MRI scoring}

Clinical assessment was performed by a board-certified rheumatologist. ${ }^{52}$ Synovitis was scored semiquantitatively analogously to RAMRIS ${ }^{33-35}$ criteria on a 0 -to-3 rating scale by three independent readers with 18 years, 9 years and 1 year, respectively, of experience in musculoskeletal imaging. Agreement of at least two readers was defined as reference standard. Additionally, we dichotomised all joints into non-inflamed (RAMRIS $=0$ ) and inflamed joints (RAMRIS $\geq 1$ ) to evaluate the potential of fractal analysis and perfusion modelling for identification of inflammation. All readers were blinded to clinical data and fractal analysis results. We evaluated six joints per patient: distal radioulnar joint, radiocarpal joint and metacarpophalangeal joints $2-5$. We did not evaluate the first metacarpophalangeal and proximal interphalangeal joints due to their peripheral location with potential imaging artefacts in quantitative measurements and we did not include the intercarpal joints due to potential difficulties in placing a region of interest (ROI) in normal cases due to partial volume effects. The distal interphalangeal joints were not included in the images.

\section{Fractal analysis}

Like the underlying self-similar and scale-invariant vascular tree, also perfusion patterns adopt a fractal organisation. ${ }^{15}$ This fractal organisation justifies the investigation of fractal analysis as a method to assess the patterns of perfusion abnormalities in inflammatory arthritis. Due to scale-invariance as a central characteristic of fractals, the examination of perfusion patterns is independent of resolution, as long as pathologically relevant regions are delineated. ${ }^{18}$

Maps of local FD were calculated as previously described. ${ }^{18}{ }^{36}$ FD was evaluated in synovial tissue of six individual joints per patient, that is, radioulnar, radiocarpal and second to fifth metacarpophalangeal joints. FD constitutes an absolute and quantitative 
parameter for vascular structure based on chaos of perfusion patterns. ${ }^{18}{ }^{36} \mathrm{FD}$ maps were calculated as follows using first-pass Mr perfusion images, which were considered two-dimensional textures with intensity as third dimension. local fractal analysis was performed by calculating maps of the local FD. Mathematically, the method is based on the blanket fractal analysis method ${ }^{37}$ and formulated for a local kernel. ${ }^{38}$ A blanket with an upper and a lower surface is moulded to the MRI texture and iteratively raised or, respectively, lowered according to the following formulae:

$$
\begin{aligned}
& u_{\varepsilon}(i, j)=\max \left\{u_{\varepsilon-1}(i, j)+1, \underset{|(m, n)-(i, j)| \leq 1}{\max } u_{\varepsilon-1}(m, n)\right\} \\
& b_{\varepsilon}(i, j)=\min \left\{b_{\varepsilon-1}(i, j)-1, \min _{|(m, n)-(i, j)| \leq 1} b_{\varepsilon-1}(m, n)\right\}
\end{aligned}
$$

$\mathrm{u}_{\varepsilon}$ and $\mathrm{b}_{\varepsilon}$ are the upper and lower surfaces, $\varepsilon$ is scale, and $\mathrm{i}, \mathrm{j}, \mathrm{m}$ and $\mathrm{n}$ are pixel coordinates. For each iteration, the area $\mathrm{A}(\varepsilon)$ of the blanket is calculated:

$$
A(\varepsilon)=\frac{\sum_{i, j}\left(u_{\varepsilon}(i, j)-b_{\varepsilon}(i, j)\right)}{2 \varepsilon}
$$

Area is bilogarithmically plotted against $\varepsilon$ and FD is obtained from the slope of a linear fit.

A map of local FD was calculated for each point in time in the first-pass perfusion imaging sequence. A representative part of the synovial tissue was selected and constituted the ROI. The mean local FD of the ROI was recorded over time and the point in time with maximum FD was subjected to statistical analysis. Quantitative values for FD approximating 2.5 indicate uncorrelated or chaotic perfusion patterns, whereas low FD values near 2.0 are found in more homogeneous perfusion patterns.

\section{Statistical analysis}

Agreement of RAMRIS of at least two of three readers served as standard of reference for statistical analysis. Group differences were assessed by Kruskal-Wallis test. Pairwise group comparisons were performed by Mann-Whitney U test with Bonferroni correction where appropriate. Linear regression of FD versus RAMRIS was performed and multiclass area under the receiver operating curve (AUC) was calculated, 95\% CIs were estimated by bootstrapping. We performed subgroup analyses in therapy-naive patients and patients with RA, and we used the Wilcoxon signed-rank test to identify whether fractal analysis results were significantly different in those subgroups. To evaluate diagnostic accuracy for identifying inflammation using fractal analysis, perfusion modelling and a combination of both, we dichotomised our dataset into non-inflamed (RAMRIS 0) and inflamed joints (RAMRIS 1-3), and we used McNemar's test to test for statistical differences in sensitivities and specificities. Statistical significance was assumed at $\mathrm{p}<0.05$. R (V.3.4.1) was used for statistical analysis.

\section{RESULTS}

Pathophysiological framework and in silico experiments

Thirty in silico phantoms were generated (figure 1). Figure 2 shows the results of fractal analysis as a function of simulated inflammatory activity. The phantoms allowed for systematic variation of ground truth for

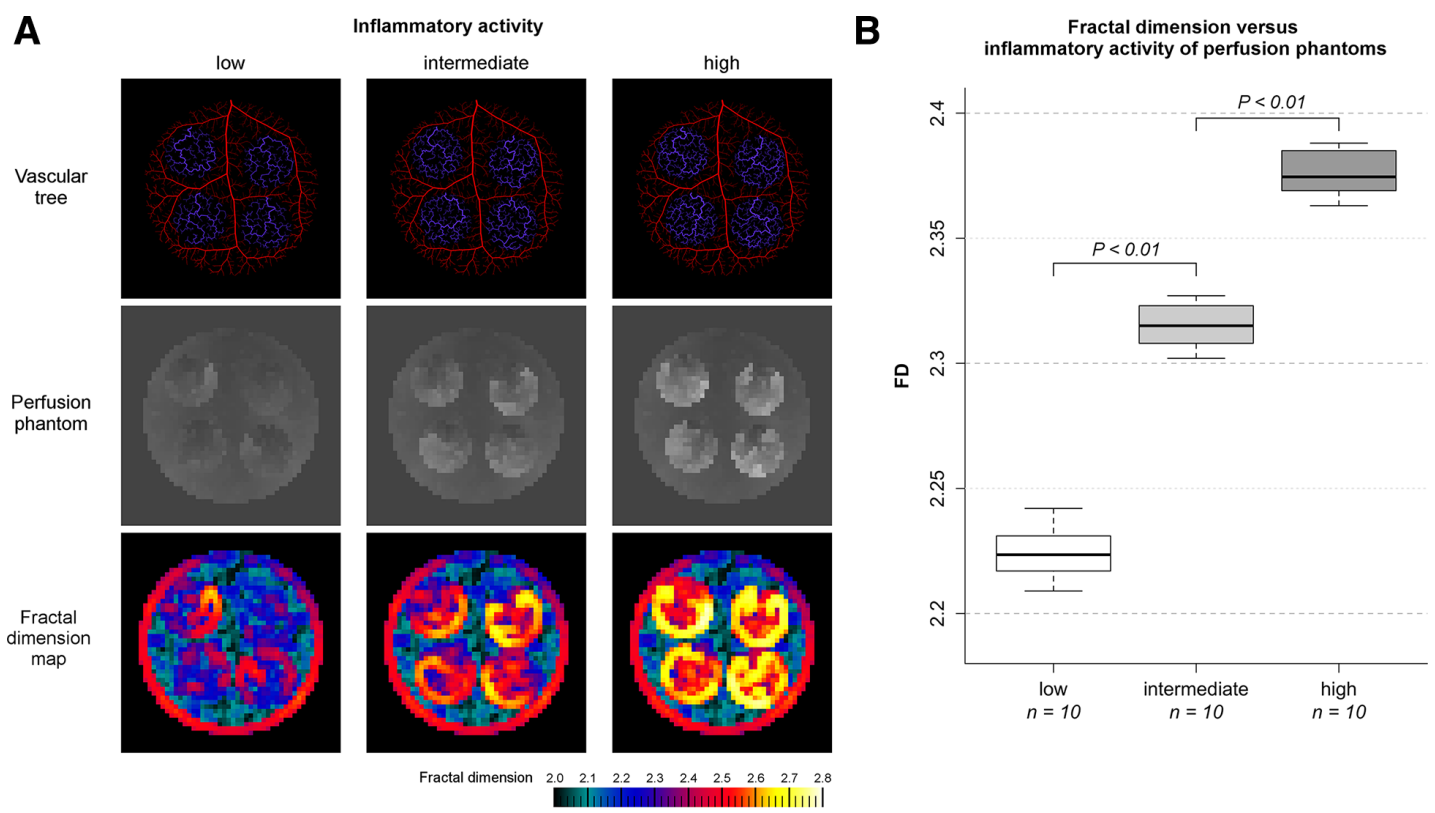

Figure 2 Fractal analysis of in silico phantoms. (A) The first row shows a model host tree with inserted angiogenic nests simulating low, intermediate and high inflammatory activity. Perfusion phantoms calculated based on vascular models are shown in the second row. Fractal analysis of perfusion phantoms yields maps of the fractal dimension (FD) encoded in the given colour scale, which are presented in the third row. (B) Boxplot of FD versus degree of simulated inflammatory activity. FD was significantly different between the three groups of inflammatory activity ( $n=10$ per group). 
Table 1 Patient characteristics and results

\begin{tabular}{llll}
\hline & Total & RA & Non-RA \\
\hline No & 36 & 24 & 12 \\
Age $(\mathrm{a})$ & $60.4(7.1)$ & $61.3(7.7)$ & $58.6(5.6)$ \\
Sex & 26 female/6 male & 17 female/7 male & Nine female/3 male \\
CRP (mg/L) & $18.6(43.2)$ & $25.0(49.5)$ & $1.8(1.8)$ \\
Symptom duration(a) & $3.6(5.1)$ & $3.1(4.8)$ & $4.6(5.7)$ \\
RAMRIS sum score & $10.2(8.7)$ & $11.6(9.6)$ & $7.4(5.6)$ \\
Fractal dimension & $2.338(0.153)$ & $2.350(0.149)$ & $2.309(0.147)$ \\
\hline
\end{tabular}

Data are presented as mean (SD), except for fractal dimension which is given as median (IQR).

CRP, C reactive protein; RA, rheumatoid arthritis; RAMRIS, Rheumatoid Arthritis MRI Scoring.

vascular structure and the resulting perfusion pattern. Fractal analysis was successfully applied to all perfusion phantoms and yielded FD a quantitative imaging biomarker of perfusion chaos. In perfusion phantoms with low, intermediate and high simulated inflammatory activity, median FD, IQR and the quotient IQR/median was 2.224 (IQR: 0.013, IQR/median: 0.006), 2.315 (IQR: 0.012, IQR/median: 0.005), and 2.375 (IQR: 0.014, IQR/ median: 0.006), respectively. FD differences were highly significant $(p<0.001)$ between each of the groups and indicated increasingly chaotic perfusion patterns with increasing grades of inflammatory activity (Spearman's $\rho=0.94, p<0.001)$.

\section{Clinical evaluation}

Thirty-six patients were analysed, and their characteristics are presented in table 1 . In total, 216 joints were included, among them 103 with signs of inflammation on MRI. Based on ACR/EULAR ${ }^{31}$ criteria, 24 patients were classified as having rheumatoid arthritis (17 negatives and 7 positives in serology). Six patients were diagnosed with finger osteoarthritis, three with peripheral spondyloarthritis/psoriatic arthritis, two with calciumpyrophosphate disease, and one with undifferentiated arthritis. Six patients were on initial therapy with corticosteroids, seven with methotrexate, three with nonsteroidal anti-inflammatory drugs and six with biologicals. Corticosteroid injection was avoided prior to MRI examination. All patients showed signs of inflammation on MRI in at least one joint.

\section{Fractal analysis}

Fractal analysis was successful in all cases and took around 5 min per patient. Figure 3A shows example cases for each
A

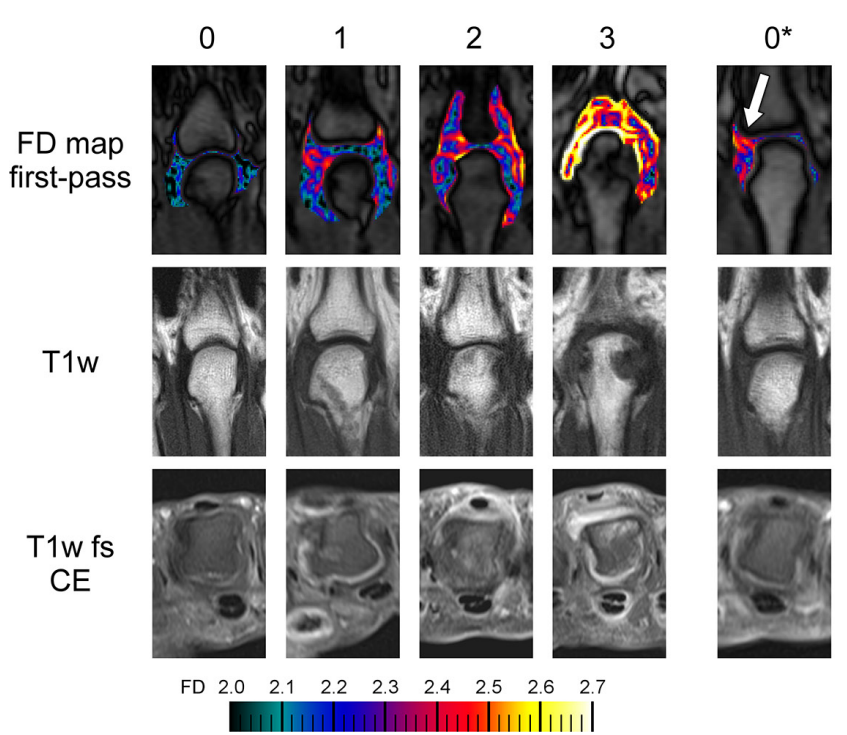

B

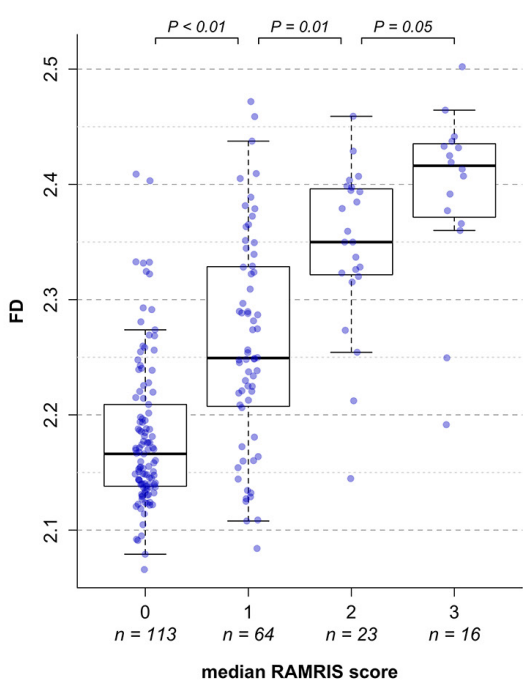

Figure 3 Fractal analysis of MRI first-pass perfusion. (A) Fractal dimension (FD) maps of the third metacarpophalangeal joint with first-pass perfusion MRI in five cases with different RAMRIS scores are presented in the first row. The second and third rows show the corresponding unenhanced T1-weighted (T1w) and contrast-enhanced T1-weighted, fat-saturated (T1w fs $\mathrm{CE})$ sequences. The patient on the far right $\left(0^{\star}\right)$ had a RAMRIS score of 0 but with an elevated fractal dimension indicating an angiogenic nest consistent with mild synovitis. (B) Boxplot of FD vs RAMRIS for all 216 joints (blue dots). Differences in median FD between the groups were statistically significant as indicated by the $p$ values given above. RAMRIS, Rheumatoid Arthritis MRI Scoring. 
of the four RAMRIS scores. Median FD (IQR) was 2.166 (0.071), $2.249(0.12), 2.350(0.075)$ and $2.416(0.06)$ in RAMRIS scores of 0 to 3, respectively, with the following optimal FD cutoffs: 2.204, 2.312 and 2.407. Figure 3B presents a boxplot of FD vs RAMRIS. The variation of FD in joints with RAMRIS scores of 0 and 1 was high with a concentration in the lower FD range in RAMRIS 0 (FD between 2.0 and around 2.2) and scattering towards higher FD. FD and RAMRIS showed good linear correlation $(\mathrm{r}=0.71, \mathrm{p}<0.01)$ and a multiclass $\mathrm{AUC}=0.84(95 \%$ CI 0.7 to 0.89$)$.

In subgroup analyses of therapy-naïve patients $(n=14)$ and patients with RA $(\mathrm{n}=24)$, fractal analysis did not show significant FD differences per RAMRIS groups with $\mathrm{p}$ ranging from 0.26 to 0.68 in therapy-naïve patients and, respectively, $0.12-0.95$ in patients with $\mathrm{RA}$.

\section{Perfusion analysis}

From perfusion analysis using the extended Tofts model, ${ }^{8}$ we obtained transfer constant $\mathrm{K}_{\text {trans }}$ and plasma volume $\mathrm{v}_{\mathrm{p}}$. Median $\mathrm{K}_{\text {trans }}(\mathrm{IQR})$ was $0.35 / 100 \mathrm{~mL}$ (IQR: 1.3), 2.26/100 mL (IQR: 5.25), 8.69/100 mL (IQR: 7.53) and $16.88 / 100 \mathrm{~mL}$ (IQR: 19.41) in RAMRIS scores 0 to 3. Median $\mathrm{v}_{\mathrm{p}}$ (IQR) was $3.4 \%$ (IQR: 7.1 ), 13.1\% (IQR: $26.3 \%$ ), $33.5 \%$ (IQR: 26.5) and $53.5 \%$ (IQR: 60.4) in RAMRIS scores 0-3.

\section{Non-inflamed versus inflamed joints}

We dichotomised our dataset to evaluate the differentiation of non-inflamed (RAMRIS 0 ) and inflamed joints (RAMRIS 1-3). FD achieved sensitivity and specificity of $83 \%(95 \%$ CI $75 \%$ to $90 \%)$ and $74 \%$ (95\% CI $65 \%$ to $82 \%$ ) for identification of inflammation, while $\mathrm{K}_{\text {trans }}$ and plasma volume $\mathrm{v}_{\mathrm{p}}$ were highly specific but not sensitive for inflammation ( $\mathrm{K}_{\text {trans }}$ : sensitivity $=59 \%$ (95\% CI $49 \%$ to $69 \%)$, specificity $=94 \%(95 \%$ CI $88 \%$ to $97 \%)$ v : sensitivity $=61 \%(95 \%$ CI $51 \%$ to $71 \%)$, specificity $=90 \%(95 \%$ CI $83 \%$ to $95 \%)$ ). When combined with fractal analysis, neither $\mathrm{K}_{\text {trans }}$ nor $\mathrm{v}_{\mathrm{p}}$ improved diagnostic accuracy over fractal analysis alone (McNemar's test) with identical sensitivity $(83 \%, 95 \%$ CI $75 \%$ to $90 \%, p=1)$ and specificity $(74 \%, 95 \%$ CI $65 \%$ to $82 \%, p=1)$ for both combinations $\left(\mathrm{FD}+\mathrm{K}_{\text {trans }}\right.$ and $\mathrm{FD}+\mathrm{v}_{\mathrm{p}}$ vs $\mathrm{FD}$ alone $)$.

\section{DISCUSSION}

The mutual and intertwined dependence of inflammation and angiogenesis constitutes the basis for the pathophysiological hypothesis tested in our study. ${ }^{1-3}$ We established fractal analysis as a novel method to quantitatively assess perfusion patterns in synovitis. Our in silico experiments validated that fractal analysis detects vascular changes due to inflammatory angiogenesis. When transferred to clinical imaging, fractal analysis strongly correlated to subjective multiparametric grading according to RAMRIS. Therefore, FD is proposed as a non-invasive, quantitative, absolute and continuously scaled imaging biomarker for assessing inflammatory angiogenesis.
Hypoxia is a central characteristic of inflamed joint tissue caused by infiltration of inflammatory cells and upregulated metabolic activity of synovial fibroblasts. ${ }^{12}$ The proinflammatory milieu increases metabolic demands and shifts vascular microenvironment towards hyperproliferation of immature vessels with unstable and chaotic structure. ${ }^{12}$ Increased vascularity, in turn, supplies endothelial surface area for further recruitment of cellular inflammatory promotors, constituting a feedback loop. Therefore, angiogenesis in the inflamed joint can be considered a route to promote cell invasion rather than efficient tissue perfusion. ${ }^{19}$ From an imaging perspective, this feedback loop can be detected by contrast-enhancement in first-pass imaging and indicates increased vascularity despite inefficient tissue perfusion. Fractal analysis of first-pass perfusion images is suitable to characterise pathological perfusion in relation to inflammatory activity.

Surprisingly, we found a number of joints with elevated FD despite RAMRIS 0. On dedicated re-evaluation of those joints, we found that fractal analysis detected discreet enhancing foci, an example joint is shown in figure $3 \mathrm{~A}$ (marked as $0 *$ ). Moreover, we correlated those joints with perfusion metrics from the extended Tofts model and we found corresponding elevation of $\mathrm{K}_{\text {trans }}$ and $\mathrm{v}_{\mathrm{p}}$, which suggests that RAMRIS has underestimated inflammation in those joints. In retrospect, we found 11 joints which displayed signs of synovitis and should have been rated as RAMRIS 1, two of them even as RAMRIS 2. However, we refrained from post hoc upscoring those joints. Therefore, with upfront RAMRIS assessment as reference, those joints constitute false-positives despite signs of inflammation on retrospective re-evaluation, which were identified with fractal analysis and perfusion analysis.

Vascular structure is currently not sufficiently accounted for in routine imaging although angiogenesis constitutes a very early pathophysiological transitioning mechanism to promote and maintain inflammation far before bone involvement. ${ }^{2}$ Our results indicate that fractal analysis provides complementary information over perfusion parameters from contrast agent kinetic models. Unlike fractal analysis, those models do not characterise microvascular structure: Consider an area with few larger vessel and an area with diffuse microvascular angiogenesisboth areas would show an increase in transfer constant and plasma volume under the assumption of flow-limited rather than permeability-limited conditions. ${ }^{8}$ In order to derive explainability of fractal analysis, our in silico experiments using software phantoms of vascular structure and perfusion allowed for a systematic variation of vascular ground truth. Previously, simulated vascular trees have been validated to accurately approximate realistic artery trees in a wide range of organ perfusion characteristics. ${ }^{26}$ Since structural assessment of the microvasculature is not routinely accessible using clinically available methods, our simulations allowed to study fractal characteristics of perfusion, and thereby, provide a proof-of-concept. 
Translating our findings to a clinical cohort with in vivo imaging, fractal analysis might help to characterise angiogenesis quantitatively and objectively in inflammatory arthritis, which might open a window of opportunity for early disease diagnosis. We see potential in cases with subclinical inflammation as hinted by some of our cases with positive fractal analysis and perfusion parameters but negative RAMRIS. Moreover, further studies should evaluate fractal analysis for treatment monitoring during antiangiogenic therapies, which are expected to normalise vascular structure, thereby prohibiting inflammatory feedback loops. ${ }^{19}$ Therefore, fractal analysis opens a perspective for further elucidating angiogenesis in inflamed joints using MRI and might eventually translate into clinical routine as a novel imaging biomarker. However, more comprehensive validation studies are needed in homogeneous clinical populations to reflect peculiarities in the underlying arthritis types. Baseline examinations in therapy-naive patients should be prospectively correlated with follow-up scans under different therapy regimens to determine the diagnostic and prognostic value of fractal analysis, as well as its potential for therapy monitoring.

This study faces the following challenges and limitations. No clinical gold standard exists to simultaneously assess changes in vascular structure and perfusion in vivo. Direct observation using arthroscopy is limited to macrovascular scales, precluding microcirculation. Histological analysis lacks three-dimensional evaluation of the architecture of microvascular networks and was not available in our study. Sonography using either Doppler imaging or contrast-enhanced techniques is limited by interobserver variability and by the lack of objective, quantitative parameters comparable to those obtained from MRI, in part due to the inability of simultaneously recording an arterial input function. Considering the above aspects, we used a single-joint four-point grading (0-3) according to RAMRIS (rather than sum scores) as reference standard for synovitis, which has been validated in RA. Our study did not include a control population with normal synovial perfusion. However, since non-inflamed synovia consists of one or two layers of cells ${ }^{1}$ perfusion analysis might be challenging. Our clinical population constituted a convenience series from a previous study, ${ }^{5}$ which featured variability both in final diagnosis and in therapeutic regimen. While this heterogeneous patient population might be considered as a strength due to reflecting a number of different clinical conditions, many proof-of-concept studies usually concentrate on a more homogeneous population. Therefore, we included subgroup analyses of both therapy-naive patients and patients with RA. Our study also included a relative high number of seronegative patients with RA, which might in part be explained by a greater need for imaging in those patients. Moreover, the small number of patients in the non-RA subgroups does not allow to draw final conclusions in these patients. However, our phantom experiments established the proof-of-concept that fractal analysis is suitable to quantitatively characterise angiogenesis patterns. Moreover, our clinical results did not suggest that diagnosis or therapeutic regimen were significant confounders with fractal analysis results. Given the high sensitivity of MRI for inflammatory lesions and relatively high number of false positives reported in the literature, ${ }^{39} \mathrm{FD}$ might identify abnormal vasculature, however, its prospective clinical yield is to be established.

Acknowledgements The authors thank Mrs. Bettina Herwig for language editing. Contributors FM conducted in silico experiments including development of the pathophysiological framework, generation of software phantoms, and conducting fractal analysis both in software phantoms and clinical imaging data. TD, STU, FP, DP and US conducted the clinical study, recruited participants and curated the patient dataset. K-GAH, TD and STU performed conventional image interpretation. FM performed fractal analysis in clinical patients and FM and MD interpreted the results. FM drafted the initial manuscript. All authors had full access to the data and take responsibility for the integrity of the data and for the accuracy of the data analysis. Additionally, all authors contributed to the interpretation of the results, and revised and approved the final manuscript. FM and TD are the guarantors.

Funding The authors have not declared a specific grant for this research from any funding agency in the public, commercial or not-for-profit sectors.

Competing interests FM holds a US patent (USPTO: 10,991,109, Patent 2021) on fractal analysis of perfusion imaging and has filed a patent application on the same topic at the European Patent Office (PCT/EP2016/071551), each together with Marc Dewey. FM receives grant support from the German Research Foundation (DFG, grant number Ml 2272/1-1 (392304398)), which covers 50\% of his position. FP has received research grants from Lilly, Novartis and UCB. FP received speaker and consultancy fees from AbbVie, AMGEN, Bristol-Myers Squibb, Hexal, Janssen, MSD, Novartis, Pfizer, Roche und UCB. K-GAH has received lecture fees from AbbVie, MSD, Novartis, and Pfizer. MD holds a United States patent (USPTO: 10,991,109, Patent 2021) on fractal analysis of perfusion imaging and has filed a patent application on the same topic at the European Patent Office (PCT/EP2016/071551), each together with Florian Michallek. MD receives grant support from the German Research Foundation for this project (DFG, grant number 392304398). MD has received grant support from the FP7 Program of the European Commission for the randomised multicentre DISCHARGE trial (603266-2, HEALTH-2012.2.4.-2). He also received grant support from German Research Foundation (DFG) in the Heisenberg Programme (DE 1361/14-1), graduate program on quantitative biomedical imaging (BIOQIC, GRK 2260/1), the Priority Programme Radiomics for the investigation of coronary plaque and coronary flow (DE 1361/19-1 [428222922] and 20-1 [428223139] in SPP 2177/1). He also received funding from the Berlin University Alliance (GC_SC_PC 27) and from the Digital Health Accelerator of the Berlin Institute of Health. MD is European Society of Radiology (ESR) Research Chair (2019-2022) and the opinions expressed in this article are the author's own and do not represent the view of ESR. Per the guiding principles of ESR, the work as Research Chair is on a voluntary basis and only remuneration of travel expenses occurs. MD is also the editor of Cardiac CT, published by Springer Nature, and offers hands-on courses on CT imaging (www.ct-kurs.de). Institutional master research agreements exist with Siemens, General Electric, Philips and Canon. The terms of these arrangements are managed by the legal department of CharitéUniversitätsmedizin Berlin. TD has received personal fees from MSD, Novartis and Canon MS.

Patient consent for publication Not applicable.

Ethics approval The study was approved by the local ethics committee of Charité-Universitätsmedizin Berlin with reference number: EA1/259/15. Participants gave informed consent to participate in the study before taking part. Provenance and peer review Not commissioned; externally peer reviewed.

Data availability statement Data are available on reasonable request. FM is willing to examine all reasonable requests for the full dataset. The data privacy committee of Charité-Universitätsmedizin Berlin will be involved in the case of query about access to data. Participants did not give consent for data sharing but the presented data are anonymised and risk of identification is low.

Open access This is an open access article distributed in accordance with the Creative Commons Attribution Non Commercial (CC BY-NC 4.0) license, which permits others to distribute, remix, adapt, build upon this work non-commercially, and license their derivative works on different terms, provided the original work is properly cited, appropriate credit is given, any changes made indicated, and the use is non-commercial. See: http://creativecommons.org/licenses/by-nc/4.0/. 
ORCID iDs

Florian Michallek http://orcid.org/0000-0002-5475-0873

Sevtap Tugce Ulas http://orcid.org/0000-0003-3871-3746

Denis Poddubnyy http://orcid.org/0000-0002-4537-6015

Fabian Proft http://orcid.org/0000-0003-4306-033X

Kay-Geert A Hermann http://orcid.org/0000-0001-6142-3814

Torsten Diekhoff http://orcid.org/0000-0003-3593-1449

\section{REFERENCES}

1 Paleolog EM. The vasculature in rheumatoid arthritis: cause or consequence? Int J Exp Pathol 2009;90:249-61.

2 Leblond A, Allanore Y, Avouac J. Targeting synovial neoangiogenesis in rheumatoid arthritis. Autoimmun Rev 2017;16:594-601.

3 Elshabrawy HA, Chen Z, Volin MV, et al. The pathogenic role of angiogenesis in rheumatoid arthritis. Angiogenesis 2015;18:433-48.

4 Cyteval C. Doppler ultrasonography and dynamic magnetic resonance imaging for assessment of synovitis in the hand and wrist of patients with rheumatoid arthritis. Semin Musculoskelet Radiol 2009;13:066-73.

5 Ulas ST, Hermann KG, Makowski MR, et al. Perfusion in hand arthritis on dynamic contrast-enhanced computed tomography: a randomized prospective study using $\mathrm{MRI}$ as a standard of reference. Skeletal Radiol 2021;50:59-68.

6 Sewerin P, Schleich C, Brinks R, et al. Assessing associations of synovial perfusion, cartilage quality, and outcome in rheumatoid arthritis using dynamic contrast-enhanced magnetic resonance imaging. J Rheumatol 2020;47:15-19.

7 Wojciechowski W, Tabor Z, Urbanik A. Assessing synovitis based on dynamic gadolinium-enhanced MRI and EULAR-OMERACT scores of the wrist in patients with rheumatoid arthritis. Clin Exp Rheumatol 2013;31:850-6.

8 Tofts PS, Brix G, Buckley DL, et al. Estimating kinetic parameters from dynamic contrast-enhanced T(1)-weighted MRI of a diffusable tracer: standardized quantities and symbols. J Magn Reson Imaging 1999;10:223-32.

9 Vordenbäumen S, Schleich C, Lögters T, et al. Dynamic contrastenhanced magnetic resonance imaging of metacarpophalangeal joints reflects histological signs of synovitis in rheumatoid arthritis. Arthritis Res Ther 2014;16:452.

10 Swaminathan V, Parkes MJ, Callaghan MJ, et al. With a biomechanical treatment in knee osteoarthritis, less knee pain did not correlate with synovitis reduction. BMC Musculoskelet Disord 2017;18:347

11 Østergaard M, Peterfy C, Conaghan P, et al. OMERACT rheumatoid arthritis magnetic resonance imaging studies. core set of MRI acquisitions, joint pathology definitions, and the OMERACT RA-MRI scoring system. J Rheumatol 2003;30:1385-6.

12 Konisti S, Kiriakidis S, Paleolog EM. Hypoxia--a key regulator of angiogenesis and inflammation in rheumatoid arthritis. Nat Rev Rheumatol 2012;8:153-62.

13 Reece RJ, Canete JD, Parsons WJ, et al. Distinct vascular patterns of early synovitis in psoriatic, reactive, and rheumatoid arthritis. Arthritis Rheum 1999:42:1481-4.

14 Szekanecz Z, Besenyei T, Szentpétery A, et al. Angiogenesis and vasculogenesis in rheumatoid arthritis. Curr Opin Rheumatol 2010;22:299-306.

15 Bassingthwaighte JB. Fractal vascular growth patterns. Acta Stereol 1992;11:305-19.

16 Michallek F, Dewey M. Fractal analysis in radiological and nuclear medicine perfusion imaging: a systematic review. Eur Radiol 2014;24:60-9.

17 Michallek F, Dewey M. Fractal analysis of the ischemic transition region in chronic ischemic heart disease using magnetic resonance imaging. Eur Radiol 2017;27:1537-46.

18 Michallek F, Huisman $\mathrm{H}$, Hamm B, et al. Prediction of prostate cancer grade using fractal analysis of perfusion MRI: retrospective proof-of-principle study. Eur Radiol 2021. doi:10.1007/s00330-02108394-8. [Epub ahead of print: 16 Dec 2021]

19 Ferrari M, Onuoha SC, Pitzalis C. Going with the flow: harnessing the power of the vasculature for targeted therapy in rheumatoid arthritis. Drug Discov Today 2016;21:172-9.

20 Koch AE. Angiogenesis as a target in rheumatoid arthritis. Ann Rheum Dis 2003;62 Suppl 2:60ii-7.

21 Schreiner W, Neumann F, Neumann M, et al. Limited bifurcation asymmetry in coronary arterial tree models generated by constrained constructive optimization. J Gen Physiol 1997;109:129-40.

22 West GB, Brown JH, Enquist BJ. A general model for the origin of allometric scaling laws in biology. Science 1997;276:122-6.

23 Bui AV, Manasseh R, Liffman K, et al. Development of optimized vascular fractal tree models using level set distance function. Med Eng Phys 2010;32:790-4.

24 Stokes KY, Granger DN. The microcirculation: a motor for the systemic inflammatory response and large vessel disease induced by hypercholesterolaemia? J Physiol 2005;562:647-53.

25 Gottlieb ME. Modelling blood vessels: a deterministic method with fractal structure based on physiological rules. [1990] Proceedings of the Twelfth Annual International Conference of the IEEE Engineering in Medicine and Biology Society, 1990:1386-7.

26 Talou GDM, Safaei S, Hunter PJ, et al. Adaptive constrained constructive optimisation for complex vascularisation processes. $\mathrm{Sci}$ Rep 2021;11:6180.

27 Schreiner W. Concepts and features of arterial tree models generated by constrained constructive optimization. Comments Theor Biol 2001;6:103-36.

28 Hess W. Das Prinzip des kleinsten Kraftverbrauches im dienste hämodynamischer Forschung. Veit \& Comp, 1913.

29 Murray CD. The physiological principle of minimum work: I. the vascular system and the cost of blood volume. Proc Natl Acad Sci U S A 1926;12:207-14.

30 Rajaram A, loussoufovitch S, Morrison LB, et al. Joint blood flow is more sensitive to inflammatory arthritis than oxyhemoglobin, deoxyhemoglobin, and oxygen saturation. Biomed Opt Express 2016;7:3843-54.

31 Aletaha D, Neogi T, Silman AJ, et al. 2010 rheumatoid arthritis classification criteria: an American College of Rheumatology/ European League against rheumatism collaborative initiative. Ann Rheum Dis 2010;69:1580-8.

32 Diekhoff T, Ulas ST, Poddubnyy D, et al. Ultra-low-dose CT detects synovitis in patients with suspected rheumatoid arthritis. Ann Rheum Dis 2019;78:31-5.

33 Bird P, Conaghan P, Ejbjerg B, et al. The development of the EULAROMERACT rheumatoid arthritis MRI reference image atlas. Ann Rheum Dis 2005;64 Suppl 1:i8-10.

34 Conaghan P, Bird P, Ejbjerg B, et al. The EULAR-OMERACT rheumatoid arthritis $\mathrm{MRI}$ reference image atlas: the metacarpophalangeal joints. Ann Rheum Dis 2005;64 Supp 1:i11-21.

35 Ejbjerg B, McQueen F, Lassere M, et al. The EULAR-OMERACT rheumatoid arthritis MRI reference image atlas: the wrist joint. Ann Rheum Dis 2005;64 Suppl 1:i23-47.

36 Michallek F, Dewey M. United States patent 10,991,109. method for characterizing perfusion abnormalities by means of fractal analysis of the interface region 2021

37 Peleg S, Naor J, Hartley R, et al. Multiple resolution texture analysis and classification. IEEE Trans Pattern Anal Mach Intell 1984;6:518-23

38 Novianto S, Suzuki Y, Maeda J. Near optimum estimation of loca fractal dimension for image segmentation. Pattern Recognit Lett 2003;24:365-74.

39 Boer AC, Boeters DM, van der Helm-van Mil AHM. The use of $\mathrm{MRI}$-detected synovitis to determine the number of involved joints for the 2010 ACR/EULAR classification criteria for Rheumatoid Arthritis - is it of additional benefit? Ann Rheum Dis 2018;77:1125-9 